\title{
Developing network adequacy standards for VA Community Care
}

\author{
Kristin M. Mattocks PhD, MPH ${ }^{1,2}$ (D) | A. Rani Elwy PhD ${ }^{3,4}$ | \\ Elizabeth M. Yano PhD, MSPH ${ }^{5,6}$ (D) | Justin Giovannelli JD, MPP \\ Michael Adelberg MA, MPP ${ }^{8}$ | Michelle A. Mengeling PhD ${ }^{9,10}$ | \\ Kristin J. Cunningham PMP ${ }^{11}$ | Kameron L. Matthews MD, JD, FAAFP ${ }^{11}$
}

\footnotetext{
${ }^{1}$ VA Central Western Massachusetts Healthcare System, Leeds, Massachusetts, USA

${ }^{2}$ University of Massachusetts Medical School, Worcester, Massachusetts, USA

${ }^{3}$ Center for Healthcare Organization and Implementation Research, Edith Nourse Rogers Memorial Veterans Hospital, Bedford, Massachusetts, USA

${ }^{4}$ Department of Psychiatry and Human Behavior, Alpert Medical School, Brown University, Providence, Rhode Island, USA

${ }^{5}$ VA Los Angeles HSR\&D Center for the Study of Healthcare Innovation, Implementation and Policy, VA Greater Los Angeles Healthcare System, Sepulveda, California, USA

${ }^{6}$ Department of Health Policy and Management, UCLA Fielding School of Public Health, Los Angeles, California, USA

${ }^{7}$ Georgetown University Health Policy Institute, Washington, District of Columbia, USA

${ }^{8}$ Faegre Drinker Consulting, Washington, District of Columbia, USA

${ }^{9}$ Center for Access \& Delivery Research and Evaluation (CADRE) and VA Office of Rural Health (ORH), Veterans Rural Health Resource Center-Iowa City (VRHRC-IC), lowa City VA Health Care System, lowa City, lowa, USA

${ }^{10}$ Department of Internal Medicine, University of lowa Carver College of Medicine, lowa City, lowa, USA

${ }^{11}$ Department of Veterans Affairs, Washington, District of Columbia, USA
}

\begin{abstract}
Objectives: To inform how the VA should develop and implement network adequacy standards, we convened an expert panel to discuss Community Care Network (CCN) adequacy and how VA might implement network adequacy standards for community care.
\end{abstract}

Data Sources/Study setting: Data were generated from expert panel ratings and from an audio-recorded expert panel meeting conducted in Arlington, Virginia, in October 2017.

Study Design: We used a modified Delphi panel process involving one round of expert panel ratings provided by nine experts in network adequacy standards. Expert panel members received a list of network adequacy standard measures used in commercial and government market and were provided a rating form listing a total of 11 measures and characteristics to rate.

Data Collection Methods: Items on the rating form were individually discussed during an expert panel meeting between the nine expert panel members and VA Office of Community Care leaders. Attendees addressed discordant views and generated revised or new standards accordingly. Recorded audio data were transcribed to facilitate thematic analysis regarding opportunities and challenges with implementing network adequacy standards in VA Community Care.

Principal Findings: The five highest ranked standards were network directories for Veterans, regular reporting of network adequacy data to VA, maximum wait time/ distance standards, minimum ratio of providers to enrolled population, and qualitative assessments of network adequacy. During the expert panel discussion with VA Community Care leaders, opportunities and challenges implementing network adequacy standards were highlighted.

Conclusions: Our expert panel shed light on priorities for network adequacy to be implemented under $\mathrm{CCN}$ contracts, such as developing comprehensive provider directories for Veterans to use when selecting community providers. Remaining questions 


\section{Correspondence}

Kristin M. Mattocks, PhD, MPH, VA Central Western Massachusetts Healthcare System, Birch Building, Room 112, 421 North Main Street, Leeds, MA 01053, USA.

Email: Kristin.Mattocks@va.gov

\section{Funding information}

This study was funded by the VA Health Services Research and Development (SDR 17-156). focus on whether the VA could reasonably develop and implement network adequacy standards given current Congressional restraints on VA reimbursement to community providers.

\section{KEYWORDS}

choice, Community Care, healthcare access, policy, Veterans

\section{What is Already Known on This Topic}

- Network adequacy measures are widely used in commercial marketplace.

- Standards vary and may be difficult to enforce.

\section{What This Study Adds}

- Recommendations from national experts regarding network adequacy standards for VA Community Care.

- How network adequacy recommendations were incorporated into VA Community Care Network contracts.

\section{$1 \mid$ INTRODUCTION}

The Veterans Access, Choice and Accountability Act (VACAA or "Choice Act") of 2014 transformed the way the Department of Veterans Affairs (VA) delivered care for Veterans. Prior to VACAA Veterans received a substantial majority of their care within the VA. VACAA allowed Veterans to receive care from non-VA provider networks if the Veteran had to wait longer than 30 days for VA care or if the Veteran lived further than $\mathbf{4 0}$ miles from their closest VA medica facility with a full-time primary care provider. Previous studies have described extensive implementation problems with the Choice Act, including underdeveloped community provider networks, barriers to care coordination, problems with communication and scheduling, and Veteran dissatisfaction. ${ }^{1-5}$

More recently, the Department of Veterans Affairs Maintaining Internal Systems and Strengthening Integrated Outside Networks (VA MISSION) Act of 2018 establishes a new permanent community care program, requiring VA to build a network of community providers through which Veterans can access care. The MISSION Act consolidates existing VA community care programs and provides more autonomy regarding utilization of community providers and more robust care coordination for Veterans using one consolidated program (the Community Care Network or $\mathrm{CCN}$ ) instead of multiple programs. ${ }^{6}$ VA has established contracts with two Third Party Administrators, Optum Public Sector Solutions, and TriWest Healthcare Alliance, to provide care to Veterans using their existing networks.

Under the MISSION Act, VA is facing new pressures to ensure Community Care Networks are meeting the needs of Veterans, and has added network adequacy standards into its CCN contracts. Network adequacy refers to the ability of a health plan to provide enrollees with timely access to a sufficient number of in-network providers, including primary care and specialty physicians, as well as other health care services included in the benefit plan of contractors. ${ }^{7}$ Historically, states have regulated commercial insurers' provider networks, but the content and scope of their regulation varied widely. ${ }^{8}$ The Affordable Care Act (ACA) was the first federal law to address this variability in the commercial insurance market by developing a baseline standard to ensure enrollees in health plans sold on the ACA insurance marketplaces have access to an adequate network of providers. ${ }^{7}$ Under the Obama administration, the Centers for Medicare and Medicaid Services (CMS) defined an adequate network as meeting two criteria: a minimum number of providers and maximum travel time and distance to those providers. ${ }^{9}$ Similarly, the US Department of Health \& Human Services (HHS) gave states with state-based insurance marketplaces substantial leeway for determining their own network adequacy, ${ }^{10}$ whereas states with federally facilitated marketplaces implemented existing state adequacy standards or relied on National Committee for Quality Assurance (NCQA) and Utilization Review Accreditation Commission (URAC) requirements. ${ }^{11}$ However, under the Trump administration, federal oversight of network plans was discontinued, and states have authority to review the networks of at least some of the plans sold in their marketplaces. In states without regulatory authority or means, third-party accreditation entities such as the NCQA oversee networks, though these organizations do not set specific network standards or remediate network deficiencies. ${ }^{12}$

There are several inherent challenges to developing a comprehensive set of network adequacy standards for VA CCN. Because enrolled Veterans have historically received a majority of their care within the VA, it is difficult to know how many of them will choose to seek community care using the CCN. The MISSON Act provides standardized eligibility criteria for community care for all enrolled Veterans, which allows eligible Veterans to choose whether they receive their care in the VA or through community providers. One of these eligibility criteria, access standards, allowed VA to set wait 
time and drive time standards via federal regulation that may potentially increase the number of Veterans eligible for community care. Consequently, it is difficult to predict the demand for community care, and thus, it is difficult to evaluate whether the community provider networks will be adequate prior to demonstrated need. Additionally, in the commercial marketplace, health plans can negotiate rates with providers and can therefore both entice providers to participate in plans based on rate schedules and enforce network adequacy standards. However, Congressional legislation mandates that community providers are reimbursed at Medicare rates with few exceptions, and therefore, the VA has little enforcement power in terms of regulating provider networks by negotiating rates. Finally, determinations of network adequacy may rely on the accuracy of the list of participating providers, which may be in flux due to updating provider information, addition of new providers, and removal of retiring providers and those no longer participating. In private networks, patients rely on lists of networked providers, commonly accessible online, to make informed decisions when selecting providers. Indeed, recent evidence suggests that consumers need access to network directories which are current, accurate, and user-friendly, ${ }^{13}$ though evidence suggests these directories are often inaccurate. $^{14,15}$

To inform how the VA should develop and implement network adequacy standards, as well as elements necessary for consumer information about networks (eg, provider directories), we convened an expert panel in October 2017 to discuss Community Care Network adequacy and how the VA might implement network adequacy standards for community care. Our expert panel meeting took place before the formal implementation of the MISSION Act and addressed questions relevant to the Act: (1) Is it possible for the VA to utilize network adequacy standards for its Community Care Network? (2) What unique considerations or challenges might the VA have in enforcing network adequacy standards for its community providers? and (3) How might VA network adequacy standards be similar or different than standards used in the private health insurance marketplace?

\section{2 | METHODS}

Three specific methods were required for addressing our research questions: (1) a literature review of network adequacy standards, (2) an expert consensus panel rating of these standards, and (3) an in-person expert panel meeting to review the standards and ratings and come to a final list of network adequacy standards to be included in VA Community Care contracts. We used an explanatory, sequential mixed methods design, ${ }^{16}$ where the in-person quantitative ratings of standards informed the qualitative discussion of the most highly ranked network adequacy standards in the expert panel meeting. This mixed methods approach was selected to determine whether qualitative data from the expert panel discussion supported the validity of the consensus panel ratings of the network adequacy standards. ${ }^{17}$

\section{1 | Identification of network adequacy standards}

Existing network adequacy standards were identified through a literature review and nominations from academic health policy experts. ${ }^{14,15,18-22}$ Our literature review focused on existing network adequacy standards used in private and government markets. We also included items that were considered important for building provider networks, such as consumer information on providers or "network directories". ${ }^{14}$ This review was completed prior to the meeting and distributed to attendees in advance in to enable a feedback process during the group sessions. We included both quantitative and qualitative measures, consumer protections, and reporting and regulation requirements. Qualitative standards give insurers flexibility to design networks for enrollees and use language such as "sufficient" and "reasonable" and may refer to "timely" access to providers. Such qualitative language can be interpreted differently in different contexts, giving regulators flexibility to address diverse markets and communities. ${ }^{14}$ Through a consensus panel, we asked experts to rate how important these items were to developing network adequacy standards for VA care and how feasible it was to implement this standard or practice for VA care (see below).

\section{2 | Panelist selection and expert consensus process}

We used a modified Delphi panel process drawn from the RAND/ UCLA Appropriateness Method ${ }^{23}$ to come to consensus on which network adequacy standards should be implemented in VA Community Care Networks to ensure Veterans are receiving timely, geographically proximate access to care. These highly structured meetings typically gather input from 9 to 12 relevant experts in 2 rounds of ratings of a series of items. ${ }^{24}$ Potential expert panelists were identified based on the literature review detailed above to identify individuals with relevant expertise in network adequacy. Known experts in network adequacy were also asked to identify other experts in the field, seeking a multidisciplinary mix of panelists representing academia and industry. We sent email invitations to prospective panelists until we successfully recruited nine panel members. The expert panel members included one clinician, health economists (2), health services researchers (3), policy analysts (2), and leaders in the insurance industry (2). Eight of the nine expert panel members were not VA employees, given that network adequacy measures were in their infancy at the time of the expert panel. Travel costs for attendance at an in-person meeting in Arlington, Virginia, were covered for each panelist.

To begin the expert panel process, panelists received emails containing key articles on network adequacy standard measures and associated characteristics used in commercial and government markets, and a prepanel rating form that asked panelists to rate the importance and feasibility of each candidate network adequacy measure. The prepanel rating form included a total of 11 measures and characteristics to rate. These 11 measures included 
TAB LE 1 Network adequacy expert panel ratings

\begin{tabular}{|c|c|c|}
\hline \multicolumn{3}{|l|}{ Results from network adequacy expert panel } \\
\hline Domain & $\begin{array}{l}\text { Importance mean rating } \\
\text { (range) }\end{array}$ & $\begin{array}{l}\text { Feasibility mean } \\
\text { rating (range) }\end{array}$ \\
\hline \multicolumn{3}{|l|}{ 1. Quantitative standards for network adequacy } \\
\hline 1.1 Minimum ratios of providers to enrolled population standards ${ }^{a}$ & $4.16(3-5)$ & $3.60(3-5)$ \\
\hline 1.2 Maximum time or distance for enrollees to travel to providers ${ }^{a}$ & $4.00(3-5)$ & $3.50(3-4)$ \\
\hline 1.3 Maximum wait times to secure an appointment & $3.86(2-5)$ & $3.00(2-4)$ \\
\hline $\begin{array}{l}\text { 1.4 Quantitative time and distance standards that mirror CMS (eg, Medicare Advantage) } \\
\text { standards }\end{array}$ & $4.00(3-5)$ & $4.00(3-5)$ \\
\hline 1.5 Prioritization of maximum wait times over time and distance standards & $2.80(1-4)$ & $3.00(2-4)$ \\
\hline \multicolumn{3}{|l|}{ 2. Assessing network adequacy using qualitative measures } \\
\hline $\begin{array}{l}\text { 2.1 Qualitative standards (eg, "sufficient" numbers and types of providers) should be } \\
\text { available as a means for sites to demonstrate network adequacy compliance }{ }^{a}\end{array}$ & $4.17(2-5)$ & $3.80(2-5)$ \\
\hline \multicolumn{3}{|l|}{ 3. Consumer (Veteran) protections } \\
\hline 3.1 Developing network directories for Veterans ${ }^{a}$ & $4.71(4-5)$ & $2.75(2-4)$ \\
\hline $\begin{array}{l}3.2 \text { Information regarding quality ratings of providers should be included in network } \\
\text { directories }\end{array}$ & $2.33(1-5)$ & $2.00(1-3)$ \\
\hline \multicolumn{3}{|l|}{ 4. Reporting and regulation } \\
\hline 4.1 Reporting of semi-annual network adequacy data by each contractor ${ }^{a}$ & $4.14(3-5)$ & $3.25(3-4)$ \\
\hline 4.2 Network adequacy data should be publicly available to VA Medical Centers & $4.00(2-5)$ & $4.25(3-5)$ \\
\hline 4.3 Network adequacy data should be publicly available to Veterans & $3.83(2-5)$ & $4.25(3-5)$ \\
\hline
\end{tabular}

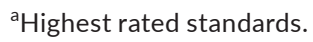

all existing private and government network adequacy measures as well as components important for network development, which fell into four major domains: quantitative network adequacy standards, qualitative network adequacy standards, network information for Veterans, and reporting and regulations requirements (Table 1).

Panelists were asked to rate each of the 11 items on both importance and feasibility: "How important is this item to developing network adequacy standards for VA care?" and "How feasible is it to implement this standard or practice for VA care?". Panelists were prompted to rate each item on a 1-5 scale of importance and feasibility where 1 = highly important/feasible and $5=$ not at all important/ feasible. A "Don't Know" box was also included. Panelists were also given the opportunity to provide comments for each item to share possible barriers or facilitators to implementing the standard, or to highlight other issues that they saw as a concern in the standard with regard to implementation with the VA health care system. Individual ratings were kept confidential. Ratings from each expert panel member were compiled, and mean ratings of importance and feasibility for each network adequacy standard were determined. The aggregated ratings comprised the consensus panel results.

\section{3 | In-person expert panel meeting}

The consensus panel results were used to guide a 6-hour expert panel in-person meeting in Arlington, Virginia, in October 2017, that brought together the nine expert panel members with senior leaders in the Office of Community Care, charged with oversight of VA Community Care and developing network adequacy standards for non-VA care. In preparation for the meeting, attendees were provided with an aggregate copy of the prepanel ratings provided by each de-identified panel member. During the meeting, the 11 items on the rating form were individually discussed, and the meeting moderator, who codesigned the modified Delphi panel process and has since applied it in over two dozen panels, ${ }^{25-27}$ guided the discussion to address discordant views and generate revised or new standards accordingly. While members of the expert panel were offered the opportunity to rerate items as outlined in a traditional Delphi approach, panel members felt that there was insufficient information available regarding VA's ability to negotiate Medicare rates and enforce network adequacy standards to provide a second rating after prolonged expert panel discussion and interaction with senior executives in VA Community Care. The panelists came to a consensus that the top five rated items were the most important regarding the future of network adequacy for VA Community Care.

The in-person meeting was recorded to capture the important interactions between expert panel members and senior officials in VA Community Care regarding the development of network adequacy standards. The meeting transcript was analyzed for major themes regarding opportunities and challenges with implementing network adequacy standards in VA Community Care. A template approach was used to code the data, ${ }^{28}$ using an a priori code list based on prior literature in network adequacy which was developed to sort and catalog key concepts for subsequent interpretation and 
analysis. Additional codes not represented in our a priori coding template and which emerged from the data were added to a finalized codebook. The transcript was coded and organized using qualitative data analysis software (ATLAS.ti Scientific Software 7.5.18), and corresponding quotations for each code were selected to identify the salient themes. Key concepts across all codes were analyzed to assess opportunities and challenges with implementing VA network adequacy standards.

\section{RESULTS}

\subsection{Expert consensus panel}

The five highest ranked standards regarding network adequacy in VA Community Care were network directories for Veterans (4.71), regular reporting of network adequacy data to VA (4.14), maximum wait time/distance standards (4.0), minimum ratio of providers to enrolled population (3.57), and qualitative assessments of network adequacy (3.57) (Table 1). These values represent preliminary ratings provided in the prepanel scores that were given after a review of network adequacy literature. However, panel members expressed reservations about developing and implementing network adequacy standards for VA Community Care given uncertainties regarding VA capacity, Medicare rates, and enforcement. Since most of the panel members were not VA employees, many of their reservations regarding implementation arose from uncertainties regarding the VA health care system and how it might be similar or different to private health care systems in organization and delivery of health care. As noted previously, senior VA leaders from VA Office of Community Care (OCC) were invited to attend the expert panel session, and thus, a conversation between non-VA network adequacy experts and VA Community Care leaders generated conversations regarding the current state of health care delivery in VA, and what would be needed to implement network adequacy standards.

A detailed qualitative analysis of some of the panel discussion between panel members and OCC leaders regarding implementing network adequacy standards is detailed below. The 11 consensus panel items corresponded to four major topics of discussion: (1) understanding VA capacity; (2) limitations in network adequacy enforcement; (3) developing appropriate qualitative standards; and (4) consumer information for Veterans.

\subsection{1 | Understanding VA capacity}

Expert panel participants noted that it would be challenging to understand whether Community Care Networks were adequate without a comprehensive understanding of each VA's need for community care. Panelists noted it was unclear how many Veterans at each facility would seek community care, as some Veterans were content to wait longer than 30 days for an appointment or were willing to travel more than 40 miles, which were the Choice Act standards at the time. One expert panel member noted the following:

First, the VA has got to figure out what its capacity is to provide services in the VA Start to measure the services that are in high demand, not the entire spectrum of services. Without understanding the true VA capacity, it makes no sense to have time and distance measures. So when you're thinking about building your network and the capacity, first of all, how many of your VA patients are going to be served in your facilities or clinics that are in a geographical area, and then how many are you going to have to send out?

One senior VA OCC leader echoed these concerns and emphasized the growth of VA health care use:

But we need to know what our needs are in the first place. And the scary part is regardless of what the assessment shows and what we've seen over Choice, all estimates may be completely grossly wrong as we've seen because I mean we grew $30 \%$ in the last fiscal year with Choice alone. That's across the board. No one estimated that we were going to give that much care. Some of these Veterans were receiving services in house. But that's just growth of VA, $30 \%$ in one year alone. It's going to be hard for our markets to estimate that, absorb it, or reflect back to our new contractors that this is what we need to purchase because it's changing.

In response, another expert panel member noted the inadequacy of standards for most facilities:

Now if the VA will provide the majority of care, then the network you're trying to manage is almost entirely on the tail. I think we know that the network adequacy standards that exist are fairly inadequate for the tail. So if we're looking for a solution that is built for the mast to apply to a situation that is almost entirely on the tail, it just seems like we're looking in the wrong place.

\subsubsection{Limitations in network adequacy enforcement}

An important point raised by several experts was how much bargaining power the VA had with contracted networks to enforce network adequacy standards. Because under the Choice Act, VA can only reimburse providers at the Medicare rate, with few exceptions, VA has little bargaining power in ensuring compliance with network adequacy standards. One member of the expert panel summarized this below: 
If VA only pays the Medicare rate for specialists, you don't have a lot of leverage to say "here are our expectations for you to see our patients". You have no leverage to hold the providers accountable, which is why I think the payment rates are what need to be fixed.

Another panel member concurred:

You don't have a lot of leverage with a lot of docs to say we, here's our time expectations for you to see our patient. And the need to be able to deviate on the as-needed basis from Medicare maximums because you're not going to get a lot of specialists at those rates. And then to say and by the way, for the privilege of getting a Medicare rate, here's all the other things we're going to have, require you to do for us. It's putting the VA in an impossible situation.

\subsection{3 | Developing appropriate quantitative standards}

Another focus of the discussion was on developing appropriate time and distance metrics for network adequacy. As one expert pointed out, with the relative dearth of subspecialty providers across the country, time and distance standards were less important given the relative paucity of specialty care providers in certain geographic areas.

I'm a little skeptical (about time and distance standards) other than in primary care. Orthopedics are all subspecialists, and so to have a metric at the orthopedic level doesn't solve your problem. That's why it's so much more nuanced and qualitative than people believe. Time and distance don't make sense for subspecialty care.

Another member summarized the concerns about developing appropriate standards:

Quantitative standards can be very helpful in promoting adequate networks, but exceptions will be needed depending on geography, rural versus metro, and provider workforce shortages. Developing capacity over time to assess adequacy of networks in light of VA patient utilizations and professional workforce supply in diverse communities will be important.

Finally, another panel member pointed out the importance of understanding quality among non-VA care providers:

Quality metrics are more important than time and distance standards. I'd be spending a lot more time trying to figure out how to get the right specialists rather than just the numbers of specialists.

\subsubsection{Consumer information for Veterans}

Expert panel participants spoke about the importance of having updated directories of available providers for Veterans. At the time of the meeting, one senior VA OCC official noted that network directories were for VA schedulers and were not yet in the hands of Veterans:

It is mostly for the VA scheduler, this directory. It's almost like an FYI for the provider. It's 'here's who we have'. But right now, Veterans have Choice. So they can ask us to credential and enroll a provider. So you need to know who we have today so you can come armed with who you want. So that's why I want it to be transparent to the Veterans or any stakeholders.

Another panel member spoke about the importance of Veterans having information about the networks and how non-VA care works.

It's health insurance literacy. And as you start getting into networks and providers, especially people who are used to the VA, just going to the VA, they may not understand. And the focus and emphasis on educating your patient population who are going to be going into the community, not just getting care at the VA, I think is important.

The accuracy of the directory is important. But is it more important for the patient or is it for the VA personnel who are navigating the system? And if it's a VA system, then I think you have different information that you don't necessarily have to present to the Veteran.

\section{4 | DISCUSSION}

To the best of our knowledge, this is the first examination of recommended network adequacy standards in VA community care. Network adequacy standards have long-served as a means to ensure that clinical networks have sufficient geographic and provider coverage to ensure that enrollees are able to get the services they need. These standards were designed for networks that represent a health care plan's entirety of in-network providers. Prior to the MISSION Act, the VA system already had a 'network' of preferred providers represented by the 1255 VA health care facilities nationwide. However, under the auspices of the Choice Act and MISSION legislation, VA contracted with external networks of providers to ensure Veterans had access to timely, geographically accessible care. 
It was these augmented networks that our expert panel was tasked with providing guidance on how to measure network adequacy. Our mixed methods analysis of expert panel members' consensus ratings and qualitative discussions of these standards determined that network standards used by health care plans could not be simply adapted by the VA health care system and shed light on priorities for network adequacy to be implemented under the new CCN contracts. The most important element identified was developing comprehensive provider directories for Veterans to use when selecting community providers, citing the need for consumers to have accurate information of the community provider networks. While experts also ranked quantitative and qualitative standards as important, the overriding focus of the expert panel meeting was whether the VA could reasonably develop and implement network adequacy standards given existing Congressional restraints on VA reimbursement to community providers.

As noted in the methods section of this paper, the expert panel took place prior to the implementation of the MISSION Act in June 2019 , but many of the network adequacy recommendations from the expert panel have been implemented in contracts with Optum Public Sector Solutions and TriWest Healthcare Alliance, the contractors selected to administer community care to Veterans (Figure 1). For example, the expert panel's highest rated domain regarding network adequacy was that it is critical for Veterans to have access to provider information in the network. Previously, VA had limited access to information regarding non-VA providers to Veterans, making it difficult for Veterans to understand the depth of the network or make choices regarding providers. Since MISSION Act implementation, VA has launched several initiatives to ensure Veterans and their VA health care providers are aware of the availability of community providers in their geographic area. Veterans may access a list of networked community providers through the online VA locator tool based on data in the Provider Profile Management System (PPMS). The locator tool allows Veterans to enter their zip code and the type of care that they would like to receive (eg, primary care, obstetrics and gynecology, mental health) and a list of providers appears that the Veteran may call to schedule an appointment. Additionally, VA's new Referral Coordination Initiative allows a VA care coordination team to capture Veterans' preferences for care (eg, geographic preferences) and for Veterans and their care team to view a list of available community providers, along with a comparison of wait times for community care compared to VA care for the type of care the Veteran requires. The PPMS system receives nightly updates from VA contractors regarding providers enrolled in the networks, though the system is not yet able to reflect whether providers are accepting new patients or not.

Expert panel members identified the importance of incorporating drive time and appointment availability standards, as well as minimum ratios of providers to enrolled members. Under the $\mathrm{CCN}$ contracts, VA has incorporated two network adequacy measures to assess contract performance: geographic accessibility (drive time) and appointment availability (wait time), which are divided into four categories of care to include medical and mental health care, dental, complementary and integrative health and pharmacy.

To address the semi-annual network adequacy reporting recommendation, contractors must report network adequacy monthly to VA, which could include a corrective action plan if the contractor identifies deficiencies in their provider network. VA evaluates the network adequacy performance standards with each contractor quarterly, which is an independent assessment by VA to determine whether the contractor is meeting standards and their monthly reports are accurate self-evaluations. Contracted providers will also be offered a quarterly provider satisfaction survey which will be reported to VA. Finally, Optum and TriWest will work with VA to

\begin{tabular}{|l|c|c|c|c|c|}
\hline \multicolumn{3}{|c|}{ Performance Objective (PO) 1: Health Services Network } \\
\cline { 2 - 6 } & Accessibility/Drive Times & \multicolumn{3}{c|}{ Appointment Availability } \\
\cline { 2 - 6 } & Primary Care & General Care & Emergent & Urgent & Routine \\
\hline Urban & 30 & 45 & $24 \mathrm{hrs}$ & $48 \mathrm{hrs}$ & 30 days \\
\hline Rural & 45 & 100 & $24 \mathrm{hrs}$ & $48 \mathrm{hrs}$ & 30 days \\
\hline Highly Rural & 60 & 180 & $24 \mathrm{hrs}$ & $48 \mathrm{hrs}$ & 30 days \\
\hline
\end{tabular}

\begin{tabular}{|l|c|c|}
\hline \multicolumn{3}{|c|}{ PO 2: Complimentary \& Integrative Health Services (CIHS) } \\
\hline & Accessibility/Drive Times & Appointment Availability \\
\hline Urban & 45 & 30 days \\
\hline Rural & 100 & 30 days \\
\hline Highly Rural & 180 & 30 days \\
\hline
\end{tabular}

\begin{tabular}{|l|c|c|c|c|c|}
\hline PO 3: Dental & \multicolumn{5}{|c|}{ Appointment Availability } \\
\cline { 2 - 6 } & $\begin{array}{c}\text { Accessibility/Drive Times } \\
\text { Dentistry }\end{array}$ & $\begin{array}{c}\text { Specialized } \\
\text { Dentistry }\end{array}$ & Emergent & Urgent & Routine \\
\hline Urban & 30 & 45 & $24 \mathrm{hrs}$ & $48 \mathrm{hrs}$ & 30 days \\
\hline Rural & 45 & 100 & $24 \mathrm{hrs}$ & $48 \mathrm{hrs}$ & 30 days \\
\hline Highly Rural & 90 & 180 & $24 \mathrm{hrs}$ & $48 \mathrm{hrs}$ & 30 days \\
\hline
\end{tabular}

FIGURE 1 Maximum drive times and appointment availability times 
develop Community Care Network Healthcare Services Network Quality and Performance Criteria to identify High Performing Providers and provide High Performing Provider quality and performance data to VA monthly. ${ }^{29}$

One concern of expert panel members was whether VA would be unable to enforce network adequacy standards without bargaining power to force networks to meet adequacy standards. Because VA generally does not reimburse above the Medicare rate, it is limited in its ability to enforce standards. Implicit in network adequacy standards is that health plans can negotiate rates with providers and can therefore entice providers to participate in plans based on rate schedules. A similar concern that was raised was with regard to the quality of the providers in the Community Care Network, and whether quality of providers was assessed in context of network adequacy standards. Though quality is not assessed as a part of network adequacy standards, Section $1703 \mathrm{C}$ of 38 USC, as added by section 104 of the VA MISSION Act of 2018, requires VA to establish standards for quality regarding hospital care, medical services, and extended care services furnished by the Department, including through non-Department health care providers in community care. ${ }^{30}$ VA has determined standards of quality to include timely care, effective care, safe care, and Veteran-centered care. ${ }^{30}$ These standards for quality were selected based on availability of comparative data for community providers and importance to Veterans. Private sector health plans report these data directly to the National Committee for Quality Assurance (NCQA) and then VA uses this information for purposes of comparing its care with that of community providers. Recent studies suggest that quality measures may be as important as access measures with regard to network adequacy, and significant differences in access and quality of providers may occur based on geographic location of care (metropolitan vs rural). ${ }^{31}$

Another major concern among the expert panel members was that it would be difficult to gauge whether networks were adequate if there was not clear evidence of the demand for non-VA care among Veterans. However, in the first year since MISSION implementation, nearly 2.2 million Veterans have received more than 5 million Community Care referrals. The top categories of community care referrals to date include emergency care (632 000 referrals), home health care (585 000), radiology (389 000), inpatient care (319 000), and dental care (246 000). In addition to these numbers, more than 250000 Veterans have utilized community-based urgent care since MISSION, as urgent care was a new benefit provided under MISSION for eligible Veterans. Taken together, these numbers suggest Veterans are receiving substantial numbers of authorizations for community care and, in the case of urgent care, are utilizing urgent care services, which don't require pre-authorization.

There are several important limitations to this study. First, the expert panel chose not to do a second round of rating of network adequacy measures due to the issues related to Medicare rates under the Choice Act and inability to assess demand for community care. Second, the expert panel meeting was conducted prior to the passage of the MISSION Act, and therefore, the experts did not have access to some of the components of the subsequent legislation. Future research should focus on understanding variations in network adequacy across contractors and in urban/rural areas of the country and should include a focus on all types of care including primary care, mental health, and specialty care. Network adequacy should also be carefully evaluated for types of care that the VA does not currently provide, such as obstetrical care, as women Veterans have no ability to get this care within VA and thus the adequacy of these networks is of particular importance. Finally, future research should evaluate Veterans use of urgent and emergency care under MISSION, as utilization of urgent care has increased substantially perhaps in lieu of utilization of community-based or VA primary care.

In conclusion, as the VA further implements the MISSION Act and the CCN contracts, it is critical that VA develops a comprehensive understanding of the demand for community care services in order to work with the contractors to develop appropriate network adequacy standards. Identifying ways to ensure Veteran access goes beyond measures of network adequacy and will require a systemswide approach where network adequacy is an essential, but not sole, component of improving access to health care.

\section{ACKNOWLEDGMENT}

Joint Acknowledgment/Disclosure Statement: This study was funded by the VA Health Services Research and Development (SDR 17-156). There are no potential conflicts that exist for any author listed on this manuscript. The opinions expressed here are those of the authors and do not represent the official policy or position of the US Department of Veterans Affairs or the U.S. government.

Disclaimer: The opinions expressed here are those of the authors and do not represent the official policy or position of the US Department of Veterans Affairs or the US government.

\section{CONFLICT OF INTEREST}

No potential conflicts exist.

\section{ORCID}

Kristin M. Mattocks (iD https://orcid.org/0000-0002-6012-3008 Elizabeth M. Yano (iD https://orcid.org/0000-0002-9385-0025

\section{REFERENCES}

1. Mattocks K, Mengeling M, Sadler A, et al. The Veteran's Choice Act: a qualitative examination of rapid policy implementation in the Department of Veterans Affairs. Med Care. 2017;55:S71-S75.

2. Mattocks K, Yano EM, Brown AN, et al. Examining women Veteran's experiences, perceptions and challenges with the Veterans Choice Program (VCP). Med Care. 2018;56(7):557-560.

3. Sayre GG, Neely EL, Simons CE, et al. Accessing care through the Veterans Choice Program: the Veteran experience. J Gen Intern Med. 2018;33(10):1714-1720.

4. Jones AL, Fine MJ, Stone RA, et al. Veteran satisfaction with early experiences of health care through the Veterans Choice Program: a concurrent mixed methods study. J Gen Intern Med. 2019;34(9):1925-1933.

5. Nevedal AL, Wagner TH, Ellerbe LS, et al. A qualitative study of primary care providers' experiences with the Veterans Choice Program. J Gen Intern Med. 2019;34(4):598-603. 
6. Kupfer J, Witmer R, Do V. Caring for those who serve: potential implications of the Veterans Affairs Maintaining Internal Systems and Strengthening Integrated Outside Networks Act of 2018. Ann Intern Med. 2018;169(70):487-489.

7. McCarty S, Farris M. ACA implications for state network adequacy standards. Robert Wood Johnson Foundation. 2013. http://www. rwjf.org/content/dam/farm/reports/issue_briefs/2013/rwjf4 07486. Accessed October 15, 2019.

8. Giovannelli J, Williams A. Regulation of narrow networks: with federal protections in jeopardy, state approaches take on added significance. To the Point, The Commonwealth Fund. 2017. https://www.commonweal thfund.org/blog/2017/regulation-narrow-networks-federal-protection s-jeopardy-state-approaches-take-added. Accessed October 9, 2019.

9. Giovannelli J, Lucia K, Corlette S. Implementing the Affordable Care Act: state regulation of marketplace plan provider networks. Issue Brief (Commonwealth Fund). 2015;10:1.

10. Giovannelli J, Lucia K, Corlette S. Regulation of Health Plan Provider Networks. Narrow networks have changed considerably under the Affordable Care Act, but the trajectory of regulation remains unclear. Health Affairs. 2016. https://www.healthaffairs. org/do/10.1377/hpb20160728.898461/full/healthpolicybrief160. pdf. Accessed October 9, 2019.

11. National Committee for Quality Assurance. Network adequacy and exchanges. Washington, DC: National Committee for Quality Assurance; 2013.

12. Giovannelli J, Lucia K. Amid market uncertainty, Trump Administration retreats from health plan oversight. Commonwealth Fund. 2017. https://www.commonwealthfund.org/blog/2017/amid-marketuncertainty-trump-administration-retreats-health-plan-oversight. Accessed October 20, 2019.

13. Hall M, Ginsburg P. A better approach to regulating provider network adequacy. Brookings Center for Health Policy. 2017. https://www. brookings.edu/wp-content/uploads/2017/09/regulatory-optionsfor-provider-network-adequacy.pdf. Accessed October 15, 2019.

14. Wishner JB, Marks J. Ensuring compliance with network adequacy standards: lessons from four states. Urban Institute. 2017. www. urban.org/sites/default/files/publication/88946/2001184-ensur ing-compliance-with-network-adequacy-standards-lessons-fromfour-states_0.pdf. Accessed June 5, 2020.

15. Haeder SF, Weimer DL, Mukamel DB. Secret shoppers find access to providers and network accuracy lacking for those in marketplace and commercial plans. Health Aff. 2016;34(7):1160-1166.

16. Fetters MD, Curry LA, Creswell JW. Achieving integration in mixed methods designs - principles and practices. Health Serv Res. 2013;48:2134-2156.

17. Creswell JW, Clark VL. Designing and Conducting Mixed Methods Research. Sage Publications (US); 2017.

18. Grant MJ, Booth A. A typology of reviews: an analysis of 14 review types and associated methodologies. Health Inform Libraries $J$. 2009;26:91-108.

19. US Centers for Medicare \& Medicaid Services. 2011 CMS Medicare Advantage Network adequacy criteria development overview. 2011. https://www.cms.gov/Medicare/Medicare-Advantage/MedicareAd vantageApps/downloads/2011_MA_Network_Adequacy_Criteria_ Overview.pdf. Accessed June 5, 2020.
20. Brodsky K, Smith B, Rodin D. Making Affordable Care Act coverage a reality: a national examination of provider network monitoring practices by states and health plans. 2015. https://www. healthmanagement.com/wp-content/uploads/HMA-Final-Repor t-RWJF-Project-Provider-Network-Monitoring-Compliance-Surve y-Oct-2015.pdf. Accessed June 5, 2020.

21. Haeder SF, Weimer DL, Mukamel DB. Narrow networks and the affordable care act. JAMA. 2015;314(7):669-670.

22. Polsky D, Cidav Z, Swanson A. Marketplace plans with narrow physician networks feature lower monthly premiums than plans with larger networks. Health Aff. 2016;35(10):1842-1848.

23. Fitch $\mathrm{K}$, Bernstein SJ, Aguilar MD, et al. The RAND/UCLA Appropriateness Method User's Manual. Santa Monica, CA: RAND Corporation; 2001.

24. Jones J, Hunter D. Consensus methods for medical and health services research. BMJ. 1995;311(7001):376.

25. Rubenstein LV, Fink A, Yano EM, et al. Increasing the impact of quality improvement on health: an expert panel method for setting institutional priorities. Joint Commission J Qual Improve. 1995;21:420-432.

26. Washington D, Yano E. Expert Panel on Determinants of Women Veterans' Use of Ambulatory Care Services. Santa Monica, CA; 2003.

27. deKleijn M, Lagro-Janssen AL, Canelo I, et al. Creating a roadmap for delivering gender-sensitive comprehensive care for women Veterans: results of a national expert panel. Med Care. 2015;53(4 Suppl 1):S156.

28. Crabtree BF, Miller WL. A template approach to text analysis: developing and using codebooks. In: Crabtree BF, Miller WL, eds. Research Methods for Primary Care, Vol. 3. Doing Qualitative Research. Sage; 1992: 93-109.

29. Department of Veterans Affairs. Reference Sheet: Network Adequacy. Office of Community Care. 2018.

30. Department of Veterans Affairs. VA standards for quality. Federal Register. 2019. https://www.federalregister.gov/docum ents/2019/10/03/2019-21538/va-standards-for-quality. Accessed June 10, 2020.

31. Haeder S. Quality Regulation? Access to high-quality specialists for Medicare Advantage beneficiaries in California. Health Serv Res Manage Epidemiol. 2019;6:1-15.

\section{SUPPORTING INFORMATION}

Additional supporting information may be found online in the Supporting Information section.

How to cite this article: Mattocks KM, Elwy AR, Yano EM, et al. Developing network adequacy standards for VA Community Care. Health Serv Res. 2021;00:1-9. https://doi. org/10.1111/1475-6773.13651 